\title{
Computer Support of Interdisciplinary Communication of Analytic Geometry and Algebra
}

\author{
Tatjana V.Kapustina, Aleksandr V.Popyrin \& Lyubov N. Savina \\ Kazan (Volga Region) Federal University, Russia
}

•Received 17 June $2015 \bullet$ Revised 22 August $2015 \bullet$ Accepted 19 September 2015

The relevance of the research is due to the modernization of higher mathematical education in Russia, which led to a significant change in the curriculum and the need to establish new relationships of disciplines. The aim of the article is to find ways to solve the problem of the interdisciplinary connections in the teaching of present course "Analytical geometry" and "Algebra" in higher education. The leading method of the study of this problem is the methodical analysis and subsequent synthesis, which allows, having analyzed the didactic content of the "Analytical Geometry" and "Algebra"courses, to identify the necessary interdisciplinary communication between them and find ways to implement them through the use of modern educational technologies. It is proved that one of the instruments for implementing these methods is a computer, in particular systems of computer mathematics (Maple, Mathematica). Article submissions may be useful to teachers of mathematical disciplines of higher education institutions.

Keywords: information learning, interdisciplinary communication, mathematics

\section{INTRODUCTION}

The curriculum of first semester students of mathematical directions at universities traditionally presents algebra and analytic geometry. From the first lessons of analytical geometry we need information from linear algebra, while algebra course begins with a different theme. Therefore, tutors of analytic geometry get the task to implement propaedeutic training elements of linear algebra. This is just one example of the need to establish interdisciplinary connections betweenalgebra and analytic geometry. The application of algebra to analytical geometry occurs at almost at every stage of learning, but also backs the use of analytic geometry concepts in algebra which gives visibility to abstract algebraic concepts.

The goal of this study - to establish the optimal interdisciplinary connections of algebra and analytic geometry. Methodology of research - the latest achievements of the theory and methodology of teaching mathematics, including - the idea of informatization of teaching mathematics (Robert, 2010; Wolfram, 2009).

Correspondence: Tatjana V.Kapustina,

Kazan (Volga Region) Federal University, 18, Kremlyovskaya Street, Kazan, 420008, Russia.

E-mail: tv_kapustina@mail.ru

doi: $10.29333 /$ iejme/300 
Interdisciplinary communication are among the key issues in the field of education (Maximova, 1988; Nagovitsina \&Sergievskii, 2014; Sergievskii, 2007). Stages of research: 2012-2013 academic years - the study of scientific and methodological literature on the topic, planning and preparation of materials of pedagogical experiment - conducting ascertaining stage of the experiment; 2013-2014 academic years - conducting formative stage of the experiment; 2014-2015 academic years the processing of the experimental results and the conclusions drawn from it, the formulation of the article. The research is supported by many years of experience of the authors as university lecturers.

\section{MATERIALS AND METHODS}

\section{Methods of pedagogical research}

To work on the issue the following methods of pedagogical research were used:

- theoretical (analysis of philosophical, psycho-pedagogical, educational, scientifically- methodical and special literature related to new means of mathematical research - computer algebra systems);

- empirical (conversations, observation and questioning of students, analysis of independent, control, training and research students' works);

- statistics (analysis of the results of the pedagogical experiment methods of mathematical statistics, their quantitative and qualitative analysis).

During the pedagogical experiment student-centered approach was carried out.

\section{Teaching methods}

Establishment of interdisciplinary connections of algebra and analytic geometry requires computer support, as modern information and communication technologies in teaching mathematics allow, on the one hand, to intensify the learning process, and on the other hand - to significantly increase the visibility of learning. In addition, there is the following argument in favor of the use of information and communication technologies: to develop modern products of computer science and methods of their use in the practice of training and future careers of graduates, these products must be used directly in the educational process of the university. This will produce a double effect: the intensification of the university learning process, and the development of elements of the methodology and techniques of informatization of mathematical research.

Goals for optimizingof the learning process of algebra and analytic geometry and the establishment of strong interdisciplinary connections of these disciplines can serve as system introduction in educational process of high school modern software - systems of computer mathematics (Maple, Mathematica, MatLab). Formulate its basic principles.

\section{The principle of expediency}

Apply computer training only when it is effective from the methodological point of view.

\section{The principle of the new challenges}

Its essence is not to transfer to the computer traditionally established techniques and methods, and to rebuild them according to the new features that the system of computer mathematics (SCM) allow. In practice, this means that during the analysis of the process of learning mathematical disciplines, losses are identified stemming from the shortcomings of its organization (lack of analysis of the content of education, insufficient establishment of interdisciplinary connections, and poor knowledge of real educational opportunities specific student groups, etc.). In accordance with the 
results of the analysis, list of tasks (training and subject) are planned, which for various reasons (large amount, huge amount of time, and so on.) cannot be solved at the moment or remain partially solved, but the solution of which is possible with the help of computer mathematics.

\section{The principle of the system approach (Afanasyev, 1980)}

It means that the introduction of SCM should be done on a systematic and methodical analysis of the group of mathematical disciplines: the structuring must be performed in order to identify sections of courses, where teaching them is appropriate to the new methods using information and communication technologies.

\section{The principle of preprogramming of support tasks}

The identified sections of courses in algebra and analytic geometry, for which a new method of using SCM is suitable as supporting tasksare useful to distinguish and program them to automate the calculations used in them (for example - basic computing tasks section of the course "Multidimensional Space" in analytic geometry: finding the intersection and unionof vector spaces, compiling equations $k$-plane defined by $(\boldsymbol{k}+\mathbf{1})$ points in an $\boldsymbol{n}$-dimensional space, and so on.).

\section{The principle of maximum clarity}

Graphics capabilities allow SCM to rebuild the traditional methods of teaching mathematics, accompanying with static and dynamic illustrations of the mathematical theory of learning that promotes deeper understanding and its assimilation by students (for example - animation showing the behavior of the graph of a polynomial based on its coefficients).

\section{The principle of maximum typing of design solutions}

This means that during the development of educational software products based on SCM, performers should strive to ensure that their proposed solutions are suited to the widest possible range of substantive problems. A characteristic feature of computer mathematics is that, along with the numerical, symbolic, visual computing and embedded programming language, they have all the tools to create educational software, and some of them are fully-fledged computer mathematical environments (e.g., Mathematica); They differ from traditional training programs and software tools, as they allow the teacher to provide training to a qualitatively higher level of use of structural and combinatorial possibilities. SCM does not necessarily require the creation of radically new (clean "computer") techniques, and suggests an organic combination of the usual forms and methods of work with new approaches and methods, creating an environment for the expansion of methodological tools of the teacher, notany sort ofbreak-up. Systems of computer mathematics have a wide range of applications, featuring invariance and modularity that allows the teacher, provided its adherence to particular principles of SCM, to realize their individual capabilities in building strategies and tactics training. Briefly it objectively describesexisting interdisciplinary communication of analytic geometry and algebra.

\section{RESULTS}

The main principles of implementation in the learning process of the university systems of computer mathematics are defined. Eight themesare revealed by requiring the establishment of interdisciplinary connections of algebra and analytic geometry, which can be optimally set by the use of computer mathematics.

\section{Analytic geometry}


It has been noted that from the beginning of training, analytic geometry requires knowledge of elements of linear algebra and an ability to perform the following calculation: solve systems of linear equations to explore up to 5 -th order (for example - the equation of the line ofthe second-orderaccording to five points), calculate determinants, to find a linear dependence of the vectors, and so on. In the study of curves and surfaces of second order information, the theory of quadratic forms is necessary.

Propaedeutic training of elements of linear algebra, carried out in the classroom for analytic geometry, it is desirable to maintain a specially prepared file. In our practice, and the conducted experiment mathematical computer system, Mathematica, is used (Kapustina, 2013).

Imagine as an example, a short program to educate students bringing augmented matrix of a linear system to echelon form; the program has three purposes at once: it calculates, displays all the steps of calculating (here manifested astraining effect), it can be used to control the "hand" of calculations (controlling effect). The design of the program is simple and obvious, it is possible to discuss with students in advance, which is useful for detailed analysis calculation algorithm for solving a system of linear equations by Gauss. The program transforms a matrix $\boldsymbol{a}$ of dimension $3 \times 4$. If necessary, it can be easily converted to any matrix. We did not use the more sophisticated programs such as, for example, GaussStepDet (Friedman \&Leora, 2010), suitable for arrays of any dimension, as their perception of the majority of first-year students is difficult because of the abundance of built-in functions of Mathematica (Wolfram, 2003, 2009).

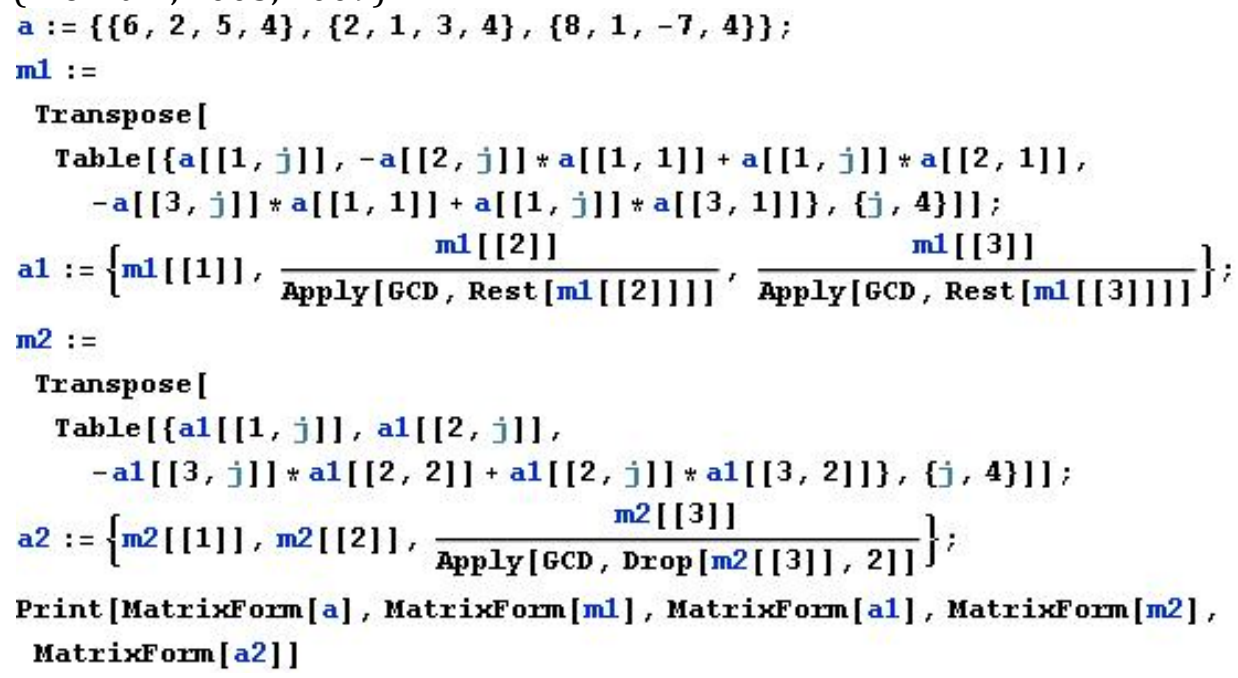

It is clearly visible four stages of reducing a matrix to echelon form via elementary transformations.For most standard linear algebra problems we do not need to program, but rather to apply the mode of computation of Mathematica.

\section{Linear algebra}

Traditionally, linear algebra, because of the abstract nature of concepts operated, is taught in the abstract (Dieudonné, 1972; Strang, 1980). The perception of these concepts will be simplified by using their geometric nature. Besides, linear algebra is of applied character, it is used in various fields of mathematics, particularly in analytic geometry (Atanasyan \& Bazylev, 2015), the statistics (Kapustina,Popyrin, \& Savina, 2014), etc. Linear algebra and analytical geometry - two sections of mathematics, 
which differ only in the language. If the geometric language is used to introduce linear algebra, it will help more clearly perceive the ideas of linear algebra, see the simplicity, accessibility, usefulness and beauty of these ideas.

\section{Systems of linear equations}

The study of linear algebra starts with solving systems of linear equations by Gauss. When solving systems of linear equations, it is very useful after the definition of solutions of linear equations, using a geometrical interpretation of the equation, to make some conclusions about the number of solutions of a system of linear equations.

Consider the system of linear equations with three unknowns over the field of real numbers. From analytical geometry, it is known that every linear equation with three unknowns is an equation of a plane. The set of solutions of equations - the intersection of all planes. Variants of the intersection of the planes known from geometry:

a) the empty set,

b) one point,

c) direct,

d) plane.

Therefore, from the algebraic point of view, the system of linear equations can be inconsistent, to have a unique solution, have an infinite set of solutions; where in case c), one free variable and two dependent, in case d) - the two free variables and one dependent. These arguments may enable students independently to extrapolate the results to the system of linear equations with more unknowns. Equivalent transformations of systems of linear equations are:

$\alpha$ ) the multiplication of the equation by a nonzero number;

$\beta$ ) addition to a single equation another equation multiplied by the number;

$\gamma$ ) adding to the system of equations (removal) of the zero equation.

Geometrically, transformations $\alpha$ ) and $\gamma$ ) do not change the audio system in the plane of the planes. Convert $\beta$ ) replaces one of the planes involved two beam planes on a plane of the beam. This fact clearly shows that the transformation of $\alpha$ ) $-\gamma$ ) do not alter the intersection of the planes. Gauss method of solution of linear equations (method of successive elimination of unknowns) at each step of using conversion b) in the case of an exception of one unknown, for example, $\mathbf{z}$, geometrically means the replacement of one of the planes from the plane of the beam of the beam parallel to the axis $\boldsymbol{O z}$. Here is an example of such illustrations for the system type:

$$
\left\{\begin{array}{c}
x-5 y+z=0 \\
3 x+7 y-z=0
\end{array}\right.
$$

Adding to the system of equations given amount (excluding unknown $\mathrm{z}$ ) leads to a system

$$
\left\{\begin{array}{l}
x-5 y+z=0 \\
3 x+7 y-z=0 \\
4 x+2 y=0
\end{array}\right.
$$

Go to (3), an equivalent system (1):

$\left\{\begin{array}{c}x-5 y+z=0 \\ 4 x+2 y=0\end{array}\right.$

Geometrical image - a beam of planes (Fig. 1). 


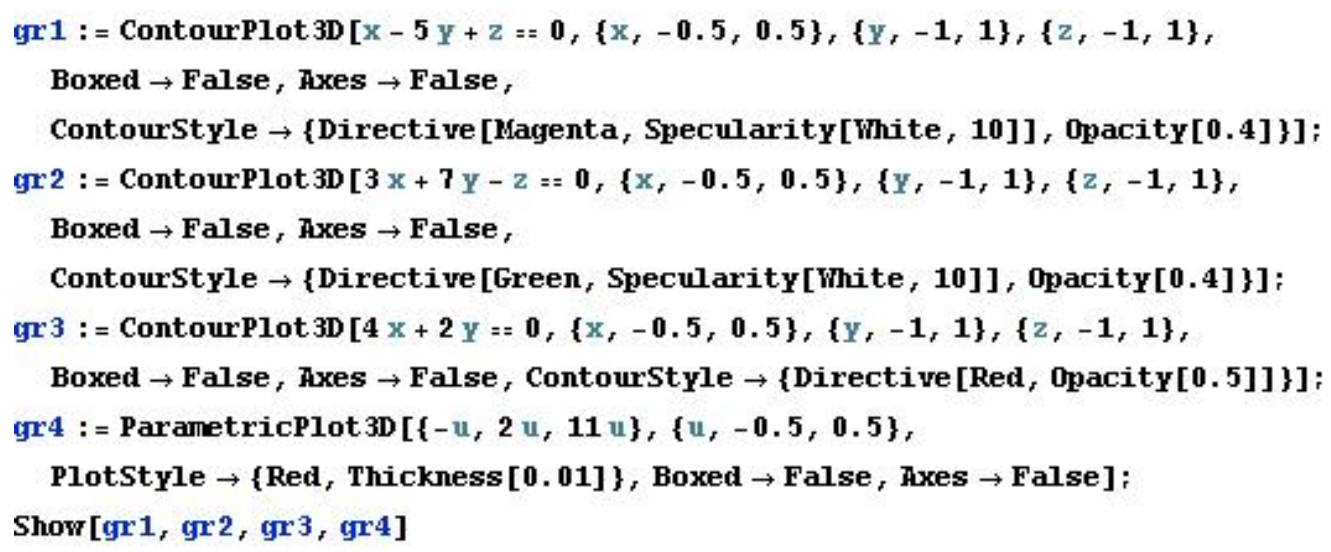

\section{Systems of linear inequalities}

The process of solving linear inequalities needs a geometric interpretationeven more. Solution of linear inequalities in two unknowns is one of the two half-planes, which divides the plane straight, the equation of which is derived from a given inequality by changing the sign inequality on the equals sign. Decision of the system of inequalities in two unknowns is the intersection of half-planes. This intersection may be an empty set, a convex polygon or infinite convex domain, partially limited by segments and rays. Students easily extrapolate these considerations on the system of inequalities with three or more unknowns. These considerations are especially important for the assimilation of the concept of a convex set, properties of the intersection of convex sets and admissible vectors of linear programming problem (Kulikov, 1979).

\section{Orthogonality}

Geometric interpretations of linearly dependent and linearly independent systems of vectors are widely known.

One-dimensional and two-dimensional vector space over the field of real numbers aregeometrically represented by a set of position vectors lying on the straight lines or planes passing through the origin. These spaces are subspaces of three-dimensional space. The concept of orthogonal projection of the vector is naturally interpreted on one-dimensional and two-dimensional space (from the end of the vector

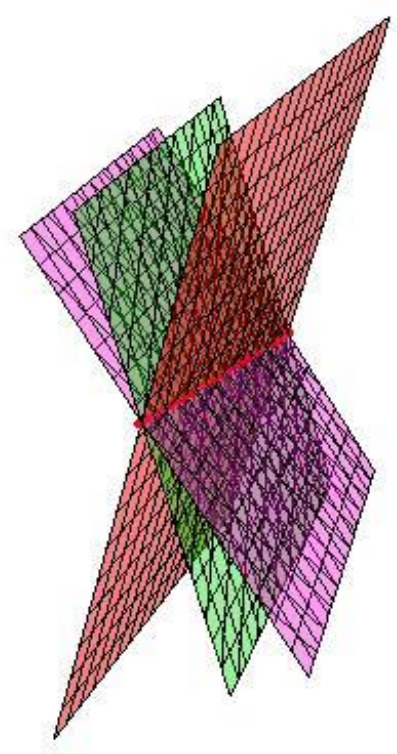

Figure 1. The beam of planes 
perpendicular to the falls straight or plane surface normal is the end of the radius vector of the orthogonal projection). If $\boldsymbol{a}-$ a vector and $\boldsymbol{b}$ - orthogonal projection of the vector $\boldsymbol{a}$ and in the space $\mathbf{V}$, then the vector $\boldsymbol{c}=\boldsymbol{a}-\boldsymbol{b}$ is called the orthogonal component. Intuitively, the vector $\boldsymbol{b}$ - "closest" to the vector $\boldsymbol{a}$ and the vector from the space $\boldsymbol{V}$. Alternatively, the vector $\boldsymbol{c}=\boldsymbol{a}-\boldsymbol{b}$ has the shortest length among all the vectors of the form $\boldsymbol{a}-\boldsymbol{d}$, where vector $\boldsymbol{d}$ from $\boldsymbol{V}$.

Orthogonal complement of space $\boldsymbol{V}$ is a subspace of $\boldsymbol{V}^{\perp}$ of all vectors orthogonal to the space $\boldsymbol{V}$ (orthogonal to all the vectors of this space). Geometrically, the orthogonal complement of the one-dimensional space (the line passing through the origin) is a plane perpendicular to the line passing through the origin; orthogonal complement of two-dimensional space - a line through the origin perpendicular to the plane of the space. By the way, from these considerations we can assume that $\left(\boldsymbol{V}^{\perp}\right)^{\perp}=\boldsymbol{V}$.

A homogeneous system of linear equations is given, for example.

$\left\{\begin{array}{l}\alpha_{11} x_{1}+\alpha_{12} x_{2}+\ldots+\alpha_{1 n} x_{n}=0 \\ \ldots \ldots \ldots \ldots \\ \alpha_{m 1} x_{1}+\alpha_{m 2} x_{2}+\ldots+\alpha_{m n} x_{n}=0\end{array}\right.$

Let $\boldsymbol{V}$ - space is generated by the row vectors of the coefficients of the unknowns $a_{1}=\left(\alpha_{11}, \ldots, \alpha_{1 n}\right), \ldots, a_{m}=\left(\alpha_{m 1}, \ldots, \alpha_{m n}\right) . \boldsymbol{L}$ - space of solutions tothe system of equations.

Then $\boldsymbol{L}=\boldsymbol{V}^{\perp}$. The reverse is true: $\boldsymbol{V}=\boldsymbol{L}^{\perp}$.

Linear systems

$\left\{\begin{array}{l}\alpha_{11} x_{1}+\alpha_{12} x_{2}+\ldots+\alpha_{1 n} x_{n}=\beta_{1}, \\ \ldots \ldots \ldots \ldots \\ \alpha_{m 1} x_{1}+\alpha_{m 2} x_{2}+\ldots+\alpha_{m n} x_{n}=\beta_{m},\end{array}\right.$

can be written in vector form:

$\left(\begin{array}{c}\alpha_{11} \\ \ldots \\ \alpha_{m 1}\end{array}\right) x_{1}+\left(\begin{array}{c}\alpha_{1 n} \\ \ldots \\ \alpha_{m n}\end{array}\right) x_{n}=\left(\begin{array}{c}\beta_{1} \\ \ldots \\ \beta_{m}\end{array}\right)$

The system of equations is consistent if and only if $\gamma_{1}, \ldots, \gamma_{n}$ are found, which

$\left(\begin{array}{c}\alpha_{11} \\ \ldots \\ \alpha_{m 1}\end{array}\right) \gamma_{1}+\left(\begin{array}{c}\alpha_{1 n} \\ \ldots \\ \alpha_{m n}\end{array}\right) \gamma_{n}=\left(\begin{array}{c}\beta_{1} \\ \ldots \\ \beta_{m}\end{array}\right)$,

i.e. column vector of free members of the system is a linear combination of the column vectors of the coefficients of the unknowns. In other words, the system of equations is consistent if, and only if, the column vector of free members belong to the space generated by the column vectors of the coefficients of the unknowns.

If the system of equations is obtained as a result of some repetitions of the experiment (the number of equations $\mathbf{m}$ is much greater than the number of unknowns $\mathbf{n}$ ), then it almost certainly has no solutions. In this case the vector $\boldsymbol{b}$ does not belong to the space columns $\boldsymbol{V}$ and Gauss method does not work. For practical purposes decision is necessary. In this case, an approximate solution is being searched. The system does not make the join due to the fact that the system is removed from the part of the "inconvenient" equations, because of which it is incompatible. In order for the system to become joint, the column-vector $\boldsymbol{b}$ vector of free members is replacedby the vector belonging to the space $V$ column vectors of the coefficients of the unknown so that an error in the calculation was the lowest for all the equations. So it is better orthogonal projection of the vector $\boldsymbol{b}$ onto the subspace of $\boldsymbol{V}$, then there is a geometric concept. Thus, you get a joint system (Strang, 1980).

\section{Complex numbers}


In the study of complex numbers algebraic and trigonometric forms of complex numbers are considered. This is due to the fact that the complex numbers are represented by points in the plane. Therefore, there is a natural desire first of all to give a geometric interpretation of operations on complex numbers. For the operations of addition, geometrical interpretation is obtained without difficulty: the addition of complex numbers is none other than the addition of vectors emanating from the origin. The subtraction operation is reduced to addition, geometrically first vector with a vector opposite the subtrahend vector which geometrical considerations is symmetrical about the origin.

Indetermining the geometric meaning of multiplication, division, exponentiation, root extraction of complex numbers using the transition from Cartesian coordinates to polar point that leads to the trigonometric form of a complex number (the module and the geometric meaning of the argument - is the distance from the point representing the number to the origin and the angle formed by the radius vector from the end point in the positive direction of the axis $\boldsymbol{o x}$, respectively. In other words, the modulus and the argument of a complex number are polar coordinates of a point representing the complex number in polar coordinate system, which terminal is at the origin, and the polar axis coincides with the positive direction of the $\boldsymbol{x}$-axis). The geometric meaning of multiplication and division: vector corresponding to the first factor is rotated by an angle equal to the argument of the second factor, and its length is stretched or compressed as many times as the length of the second vector, or, in the language of geometry, multiplication of complex numbers is the consistent application of two transformations: rotation and homothety with the center at the origin and coefficient equal to the number of the second module. Geometrically interpreted and root extraction operation the $\mathrm{n}$-th degree of complex numbers, and in the trigonometric form, the roots can be removed for any $\mathbf{n}$, and algebraically, at best, can only remove the roots of the second degree. All the roots of the $n$-th degree of non-zero complex numbers lie at the vertices of a regular $n$-gon with center at the origin, the radius of the circle is equal to the root of the $n$-th degree of a given module of a complex number. The radius vector of one of the vertices of the polygon forms an angle with the positive direction of the axis $\boldsymbol{O x}$ equal to the n-th argument of the given number (Abramov,Vilenkin, \& Dorofeev, 1980; Skopets, 1990). n-th roots of unity (as a set of points on the unit circle) under the operation of multiplication give a visual representation of a finite cyclic group.

\section{Vector product}

In studying the properties of the algebra (set with the set operations on them) vector multiplication of vectors is a perfect illustration of the non-commutative algebraic operation. In addition, this operation is distributive with respect to addition of vectors and has the property of anticommutativity:

$\boldsymbol{a} \times \boldsymbol{b}=-\boldsymbol{b} \times \boldsymbol{a}$.

In studying four-dimensional quaternion, algebra multiplication of quaternions is easier to remember, using the scalar and vector product (Faddeev, 2004; Vatulyan, 1999).

Imagine each of the two quaternions $z_{1}=a_{1}+b_{1} i+c_{1} j+d_{1} k, z_{2}=a_{2}+b_{2} i+c_{2} j+d_{2} k$ as the sum of a real number, and a three-dimensional vector: $z_{1}=a_{1}+b_{1} i+c_{1} j+d_{1} k=$ $\mathrm{a}_{1}+\mathrm{v}_{1}, \mathrm{z}_{2}=\mathrm{a}_{2}+\mathrm{b}_{2} \mathrm{i}+\mathrm{c}_{2} \mathrm{j}+\mathrm{d}_{2} \mathrm{k}=\mathrm{a}_{2}+\mathrm{v}_{2}$. Then the product of the quaternion can be represented as:

$z_{1} z_{2}=\left(a_{1}+b_{1} i+c_{1} j+d_{1} k\right)\left(a_{2}+b_{2} i+c_{2} j+d_{2} k\right)=\left(a_{1} a_{2}-b_{1} b_{2}-c_{1} c_{2}-d_{1} d_{2}\right)+\left(a_{1} b_{2}+b_{1} a_{2}\right.$ $\left.+c_{1} d_{2}-d_{1} c_{2}\right) i+\left(a_{1} c_{2}+c_{1} a_{2}-b_{1} d_{2}+d_{1} b_{2}\right) j+\left(a_{1} d_{2}+d_{1} a_{2}+b_{1} c_{2}-c_{1} b_{2}\right) k$ or $\mathrm{z}_{1} \mathrm{z}_{2}=\left(\mathrm{a}_{1}+\mathrm{v}_{1}\right)\left(\mathrm{a}_{2}+\mathrm{v}_{2}\right)=\mathrm{a}_{1} \mathrm{a}_{2}+\mathrm{a}_{2} \mathrm{v}_{1}+\mathrm{a}_{1} \mathrm{v}_{2}+\mathrm{v}_{1} \mathrm{v}_{2}=\mathrm{a}_{1} \mathrm{a}_{2}+\mathrm{a}_{2} \mathrm{v}_{1}+\mathrm{a}_{1} \mathrm{v}_{2}-\mathrm{v}_{1} \mathrm{v}_{2}+\mathrm{v}_{1} \times \mathrm{v}_{2}$, where $\mathrm{v}_{1} \mathrm{v}_{2}=$ $\mathrm{b}_{1} \mathrm{~b}_{2}+\mathrm{c}_{1} \mathrm{c}_{2}+\mathrm{d}_{1} \mathrm{~d}_{2}-$ scalar product, and 


$$
\begin{gathered}
\left.\mathrm{v}_{1} \times \mathrm{v}_{2}=\left|\begin{array}{ccc}
i & j & k \\
b_{1} & c_{1} & d_{1} \\
b_{2} & c_{2} & d_{2}
\end{array}\right|=\left|\begin{array}{ll}
c_{1} & d_{1} \\
c_{2} & d_{2}
\end{array}\right| i-\left|\begin{array}{ll}
b_{1} & d_{1} \\
b_{2} & d_{2}
\end{array}\right| j+\left|\begin{array}{ll}
b_{1} & c_{1} \\
b_{2} & c_{2}
\end{array}\right| k=\mathrm{c}_{1} \mathrm{~d}_{2}-\mathrm{d}_{1} \mathrm{c}_{2}\right) \mathrm{i}-\left(\mathrm{b}_{1} \mathrm{~d}_{2}+\mathrm{d}_{1} \mathrm{~d}_{2}\right) \mathrm{j}+\left(\mathrm{b}_{1} \mathrm{c}_{2}-\right. \\
\left.\mathrm{c}_{1} \mathrm{~b}_{2}\right) \mathrm{k}-\text { vector product. In the particular case when } \mathrm{a}_{1}=\mathrm{a}_{2}=0, \\
\mathrm{z}_{1} \mathrm{z}_{2}=\left(\mathrm{b}_{1} \mathrm{i}+\mathrm{c}_{1} \mathrm{j}+\mathrm{d}_{1} \mathrm{k}\right)\left(\mathrm{b}_{2} \mathrm{i}+\mathrm{c}_{2} \mathrm{j}+\mathrm{d}_{2} \mathrm{k}\right)=-\mathrm{v}_{1} \mathrm{~V}_{2}+\mathrm{v}_{1} \times \mathrm{v}_{2} .
\end{gathered}
$$

\section{Square forms}

To simplify the equation of the second order, given by the equation

$$
a_{11} x^{2}+2_{12} x y+a_{22} y^{2}+2 a_{13} x+2 a_{23} y+a_{33}=0
$$

in a rectangular coordinate system, the coordinate system is rotated first, so that the equation of the curve in the new coordinate system is given by

$$
a_{11}^{\prime} x^{2}+a_{22}^{\prime} y^{2}+2 a_{13}^{\prime} x+2 a_{23}^{\prime} y+a_{33}^{\prime}=0
$$

Areas of new axes of the rectangular coordinate system defined by the eigenvectors of the symmetric matrix

$$
\left(\begin{array}{ll}
a_{11} & a_{12} \\
a_{21} & a_{22}
\end{array}\right)
$$

this curve (these vectors are called the principal directions of the curve). Further, with a parallel transfer of the new coordinate system (allocation of squares), the equation of the curve takes the form $a_{11}^{\prime \prime} x^{2}+a_{22}^{\prime \prime} y^{2}+a_{33}^{\prime \prime}=0$

(The second and third equations using variables to record the same letter).

From the last equation we obtain the canonical equation of the curve and determine its type. Similar arguments allow to bring the equation of the second order to the canonical form. These arguments give a geometric interpretation of the concept of eigenvector matrix. From the geometric point of view, because the real symmetric matrix of $\mathrm{n}$ - order has $\mathrm{n}$ linearly independent eigenvectors, the conversion of a vector space with the help of such a matrix can be seen as a transition to a new basis in the vector space. The quadratic form in two variables can be considered as the left side of the equation of a quadratic curve. The canonical form of the quadratic form corresponds to a special case of the canonical equation of the curve of the second order. Similar arguments reduce the problem of reduction to the canonical form of the quadratic form of three variables to the task of bringing the corresponding equation of the second order to the canonical form (Alexandrov \&Netsvetaev, 2010; Ilyin \&Pozniak, 2009; Maltsev, 2009).

\section{DISCUSSIONS}

An analysis of the scientific and methodological literature on the topic of research has shown that the questions of inter-subject relations of disciplines of Mathematics were considered only fragmentary and from the point of view of common positions, and in particular interdisciplinary communications algebra and analytic geometry in terms of computer support systems by means of computer mathematics-were not considered by anyone. Thus, we can state that methods of the interdisciplinary connections of algebra and analytic geometry by the system implementation in the educational process of computer mathematics in the previous studies were not considered.

\section{CONCLUSION}


To test the effectiveness of methods of application of interdisciplinary connections in teaching, students of the 1st course algebra and analytic geometry, using Mathematica, two tests in each of these disciplines were conducted. The first pilot works were carried out at the beginning of the courses in order to assess the launch readiness of students of the control and experimental groups for training. The second control work was carried out at the end ofthe courses for the study and analysis of the results of the learning outcomes. Theywere solved by 32 students in the experimental group (where classes were taught at the experimental procedure), and 36 studentsin the control group (in which classes were conducted according to traditional methods).

The main purpose of the experiment was to test the pedagogical set hypothesis about the effectiveness of teaching methods using interdisciplinary connections in a computer support.

Comparison of formation of competences of students in algebra and analytic geometry at the beginning of the course revealed no differences between the study groups. Installed results were checked for statistical significance using the Student ttest. During the processing of the experiment results obtained $t_{\mathrm{e}} \approx 0.0574$, which does not exceed $t_{c} \approx 2.71$ at a significance level $\alpha=0.05$. Therefore, the null hypothesis of the equality of starting performance levels in both groups was adopted. The comparative analysis of the level of development of knowledge and skills of students in algebra and analytic geometry at the end of the course revealed the existence of differences between the study groups. The obtained value of the Student's t-test after treatment with the experimental results $t_{\mathrm{e}} \approx 5.5202$, which exceeds $t_{c} \approx 2.71$ for a significance level of $\alpha=0.05$.

Therefore, a higher level of achievement in algebra and analytic geometry in the experimental group is statistically significant and is the result of the experimental teaching methods with the use of interdisciplinary connections in a computer support using the medium of Mathematica.

The carried out experiments showed the effectiveness of the methods of interdisciplinary connections of algebra and analytic geometry. Progress in the experimental group at both subjects were higher than in the controls. A side effect was an increase in the degree of learning and motivation of students.

\section{ACKNOWLEDGMENTS}

The work is performed according to the Russian Government Program of Competitive Growth of Kazan Federal University.

\section{REFERENCES}

Abramov, A.M., Vilenkin, N.Y.,\& Dorofeev, G.V. 1980). Selected topics of mathematics. Moscow, Education.

Afanasyev, V.G. (1980). Consistency and society. Moscow, Politizdat.

Alexandrov, A.D.,\& Netsvetaev, N. (2010). Geometry. St. Petersburg, BHV-Petersburg.

Atanasyan L.S.,\& Bazylev V.T. (2015). Geometry. Part 1. Moscow, Knorus.

Dieudonné, J. (1972). Linear algebra and elementary geometry. Moscow, Nauka.

Faddeev,D.K. (2004). Lectures on algebra. (2nd ed.). St. Petersburg, Lanj.

Friedman, G.M.,\& Leora, S.N. (2010). Mathematics \&Mathematica. Selected problems for select students. St. Petersburg.

Ilyin, V.A.,\& Pozniak, E.G. (2009). Analytic geometry. Moscow, Fizmatlit.

Kapustina, T.V. (2013). Differential geometry in environment Mathematica. Saarbrucken, 180.

Kapustina, T.V., Popyrin, A.V.,\& Savina, L.N. (2014). Statistics and Econometrics from the Point of View Methodology of Mathematics. World Applied Sciences Journal,31(6), 1168-1172.

Kulikov, L.Y. (1979). Algebra and Number Theory. Moscow, Higher School.

Maltsev, A.I. (2009). Fundamentals of linear algebra. St. Petersburg, Lan. 
Maximova, V.N (1988). Interdisciplinary communication in the learning process. Moscow, Education.

Nagovitsina, O.A.,\& Sergievskii, V.V. (2014). Approach to problems of interdisciplinary education. Procedia - Social and Behavioral Sciences, 128, 489 - 492.

Robert, I.V. (2010). New information technologies in education: didactic problems; prospects. Moscow, RAO.

Sergievskii,V.V. (2007). Modernization of education and Russian. Economic Strategies, 4, 114.

Skopets,Z.A. (1990). Geometric miniatures. Moscow, Education.

Strang, G. (1980). Linear Algebra and its Applications. Moscow, Mir.

Vatulyan, A.O. (1999). Quaternions. Soros Educational Journal, 5, 117-120.

Wolfram, S. (2003). The Mathematica Book. Fifth Edition. Mathematica Version 5. - Wolfram Media / Cambridge University Press, 1301.

Wolfram,S. (2009). Mathematica Documentation Center. Wolfram Research, Date Views 07.03.14 reference.wolfram.com/mathematica/guide/Mathematica.html. 